\title{
A Grid-based Binary Model for Coulomb Collisions in Plasmas
}

B. I. Cohen, A. M. Dimits, D. J. Strozzi

March 8, 2012

Journal of Computational Physics 
This document was prepared as an account of work sponsored by an agency of the United States government. Neither the United States government nor Lawrence Livermore National Security, LLC, nor any of their employees makes any warranty, expressed or implied, or assumes any legal liability or responsibility for the accuracy, completeness, or usefulness of any information, apparatus, product, or process disclosed, or represents that its use would not infringe privately owned rights. Reference herein to any specific commercial product, process, or service by trade name, trademark, manufacturer, or otherwise does not necessarily constitute or imply its endorsement, recommendation, or favoring by the United States government or Lawrence Livermore National Security, LLC. The views and opinions of authors expressed herein do not necessarily state or reflect those of the United States government or Lawrence Livermore National Security, LLC, and shall not be used for advertising or product endorsement purposes. 


\section{A Grid-based Binary Model for Coulomb Collisions in Plasmas}

Bruce I. Cohen, Andris M. Dimits, and David J. Strozzi

Abstract-Both binary and grid-based Langevin equations models for Coulomb collisions are used in particle simulation of plasmas. We introduce a variant of the conventional binary collision algorithm for performing Coulomb collisions. In this algorithm particles in a configuration space cell are not paired for collisions. Instead, for every test particle in the cell, a unique field particle is defined by randomly sampling a velocity distribution defined on the grid by accumulating moments of the particle distribution function(s). The test and field particle pair then undergoes a collision using the standard methodology for binary collisions. The performance of the new algorithm is illustrated in example computations and compared to a drag-diffusion Langevin equations algorithm. The grid-based algorithms do not conserve momentum and energy, although with good particle statistics the non-conservation is relatively small. Conservation can be restored after collisions using a shift and scaling of the momenta. The comparative merits of the new algorithm are discussed.

Index Terms — collision processes, plasmas, particle collisions, algorithms, computer applications.

Manuscript received March 2012. This work was performed under the auspices of the U. S. Department of Energy by the Lawrence Livermore National Laboratory under Contract DE-AC52-07NA27344 and under Grant DE-FG0205ER-25710 at University of California at Los Angesles under the Multiscale Initiative program supported by the DOE Office of Scientific Computing Research.

B. I. Cohen is with the Lawrence Livermore National Laboratory, P.O. Box 808, Livermore, CA 94551. (phone: 925-422-9823; fax: 925-423-3484; e-mail: cohen1@1lnl.gov)

A. M. Dimits, and David J. Strozzi are with the Lawrence Livermore National Laboratory, P.O. Box 808, Livermore, CA 94551. (e-mail: dimits1@1lnl.gov and strozzi2@1lnl.gov) 


\section{Introduction}

In this work we introduce a new variant of a well-known algorithm for including Coulomb collisions in particle simulations of plasmas using finite-sized particles and deposition of charge and current densities onto a grid (particle-in-cell simulation, i.e., PIC simulation). In the binary algorithm, particles in a subdomain, e.g., a cell, are grouped into discrete pairs of interacting particles such that the relative velocity is scattered through an angle whose statistical variance is dictated by the theory of Coulomb collisions in a plasma, in the Fokker-Planck limit [1,2]. The post-collision velocities of the interacting pair conserve momentum and energy relative to the pre-collision velocities. In the second type of algorithm, the collisions are modeled by defining test and field particles; and the test-particle velocity is subject to drag and diffusion in three velocity dimensions using Langevin equations whose drag and diffusion coefficients depend jointly on the velocity of the test particle and the moments of the field-particle velocity distribution deposited on the configuration-space mesh $[3,4,5,6,7]$. The grid-based Langevin equations model conserves particle number trivially and conserves energy and momentum approximately in a statistical sense after averaging over many collisions and over the velocity distribution functions, although energy and momentum conservation can be repaired by scaling and shifting velocities after the Monte Carlo collisions occur on each time step [4,6]. The drag and diffusion coefficients are derived from the classical theory of screened Coulomb collisions in the Fokker-Planck limit $[4,8,9,10]$.

Coulomb collisions are an important physics component of many plasma phenomena. Furthermore, computing collisions in particle-in-cell plasma simulation is a significant computational burden; and some collision algorithms are favored over others depending on considerations of the physics and the computational burden. Thus, it is of practical interest to assess the efficacy and performance of a new variant of the standard binary collision algorithm in which the field particle is created by random sampling from a velocity distribution that is computed from statistical moments of the particles accumulated onto the configuration-space grid. In particular, we introduce a grid-based variant of the algorithm of Takizuka and Abe (TA) [1]. We describe the algorithm in Sec. II. We illustrate its performance in example calculations, examine how good the energy conservation is as a function of the particle statistics, discuss how momentum and energy conservation can be restored, and compare the new algorithm to the drag-diffusion Langevin equation algorithm of Lemons, et al [6] in Sec. III. We discuss the comparative merits of the new grid-based collision algorithm and its possible extension in Sec. IV. The merits of the grid-based TA algorithm are as follows: the grid-based TA algorithm is slightly more accurate than the Lemons, et al algoirhm in our example simulations, allows a $\sim 10 \times$ larger time step for accurate electron-ion collisions, 
and tolerates the rare large Monte Carlo kick much better than does the Lemons, et al algorithm; in situations where the particles have unequal weights so that application of the traditional TA algorithm does not conserve energy and momentum algebraically, the grid-based TA scheme is a natural alternative and energy/momentum conservation can be restored; the grid-based TA scheme, like other grid-based collision algorithms, is well suited for including Coulomb collisions in a particle-fluid hybrid plasma simulation; the grid-based TA scheme can accommodate some of the self-consistent evolution of the field-particle velocity distribution without having to solve for the Rosenbluth potentials (an elliptic equation in velocity space) to update the drag-diffusion coefficients in the Langevin equations approach of [4], [5], and [6]; the gridbased collision algorithms in [4], [5], and [6] can be subject to a noise-induced instability, while the gridbased TA scheme is not; and the grid-based TA collision algorithm can be extended straightforwardly to different collision models.

\section{Grid-based Takizuka and Abe collision algorithm}

Reference [11] gives an overview of some of the fundamentals of two Langevin equations collision algorithms and Takizuka and Abe's binary collision algorithm. In the Langevin equation models [3, 4, 5, 6, 7, 10], Langevin equations in three velocity dimensions containing drag and diffusion terms are integrated, typically with a simple first-order, forward Euler integration [3-7,10,11,14]. The algorithms are based on the theory describing screened Coulomb collisions in the Fokker-Planck limit [8, 9, 15].

In the classic work of Takiziuka and Abe [1] an algorithm for binary Coulomb collisions was introduced. In the binary algorithm, equally weighted particles in a cell are paired; and then the relative velocity vector of the two particles is scattered through a random angle with variance dictated by the theory of screened Coulomb collisions in a plasma [8,9]. After the relative velocity vector is scattered, the two scattered particle velocities are reconstructed such that particle momentum and energy are conserved algebraically. Particle number is conserved identically. Nanbu [2] extended the algorithm of Takizuka and Abe [1] to allow for a larger time step by aggregating multiple collisions. In Takizuka and Abe [1], the relative velocity of a pair is scattered through an angle $\Theta$ with variance related to

$$
\begin{aligned}
& \left\langle\delta^{2}\right\rangle=2 \pi q_{1}^{2} q_{2}^{2} n_{(1,2)<} \ln \Lambda \Delta t / m_{12}^{2} u^{3}, \\
& u=\left|\mathbf{v}_{1}-\mathbf{v}_{2}\right|, m_{12}=m_{1} m_{2} /\left(m_{1}+m_{2}\right), \delta \equiv \tan \frac{\Theta}{2} \\
& \mathbf{v}_{1}^{t+\Delta t}=\mathbf{v}_{1}^{t}+\frac{m_{12}}{m_{1}} \Delta \mathbf{u}, \quad \mathbf{v}_{2}^{t+\Delta t}=\mathbf{v}_{2}^{t}-\frac{m_{12}}{m_{2}} \Delta \mathbf{u},
\end{aligned}
$$


and through a random angle $\phi$ about the axis of the relative velocity before the scattering event, where $\ln \Lambda$ is the Coulomb logarithm [8,9]. The post-collision velocity vectors of the scattered pair are constructed from the scattered relative velocity vector. There is no separation of test and field particles in the binary scheme, and there is no assumption that the velocity distribution is isotropic and Maxwellian (ignoring corrections to $\ln \Lambda$ ). This method conserves particle number, energy and momentum.

In the work of Wang, et al.,[12] the convergence properties of the Takizuka and Abe, and the Nanbu binary collision operators with respect to particle number and time step were studied. It was found that the Nanbu collision algorithm achieved a factor of two improvement in relative accuracy over the Takizuka and Abe basic algorithm for the same time step. The underlying properties of the Nanbu algorithm were studied analytically in the work of Dimits, et al. [13].

Both the grid-based Langevin equations and binary collision algorithms can be generalized to semirelativistic collisions [16,17]. To accommodate a minority component of relativistic particles in a plasma that is otherwise non-relativistic, the binary algorithm Eq.(1a) is modified by replacing $n_{(1,2)<}$ with $n_{2}$ and replacing the denominator $m_{12} u^{3}$ with $p^{2} \mathrm{v}$ where $p$ and $\mathrm{v}$ are the momentum and velocity of the test particle in the rest frame the field particle [18]. Then

$$
\left\langle\Delta \theta^{2}\right\rangle=8 \pi q_{1}^{2} q_{2}^{2} n_{2} \ln \Lambda \Delta t / p_{1}^{2} \mathrm{v}_{1}
$$

is the variance of the polar scattering angle of the relative velocity in the rest frame of the field particle. Lorentz transformations are used between the laboratory and rest frames of the field particle as in Chapter 12 of Jackson's book [18]. We note that $n_{2}$ (the density of the field particles) is not computed relativistically here, which restricts the applicability of the model. The field particles on average must be nonrelativistic in their motion with respect to the laboratory frame. In addition, there can be modifications to the computation of $\ln \Lambda$; we return to this issue in Sec. IV. Nevertheless, this semi-relativistic model is useful in many situations of interest. A summary of the relativistic modifications to the non-relativistic TA algorithm in Eq.(1) are given in the Appendix.

We transform the binary collision algorithm of Takizuka and Abe (TA) (or Nanbu's extension to somewhat larger time steps) into a grid-based scheme as follows. Each test particle in a cell collides on a distinct field particle. To define the field particle we accumulate the lowest velocity moments of the threedimensional (3D) velocity distribution of field particles in each cell using nearest grid-point interpolation [19]. Using a 3D Cartesian representation for the velocity distribution function, we define the field-particle velocity (or momentum) in each dimension independently by randomly sampling the cumulative normalized $1 \mathrm{D}$ velocity distribution:

$$
\mathrm{v}_{\mathrm{z}}=\sqrt{2} \mathrm{v}_{\mathrm{th}, \mathrm{f}} \cos \left(2 \pi \operatorname{ranf}_{1}\right) \sqrt{-\ln \left(1-\operatorname{ranf}_{2}\right)}+\left\langle\mathrm{v}_{\mathrm{z}}\right\rangle_{\mathrm{f}}
$$


where $\mathrm{v}_{\mathrm{th}, \mathrm{f}}$ is the root-mean-square thermal velocity and $\left\langle\mathrm{v}_{\mathrm{Z}}\right\rangle_{\mathrm{f}}$ is the mean drift for the $\mathrm{v}_{\mathrm{z}}$ distribution. ranf $\mathrm{f}_{1}$ and $\operatorname{ranf}_{2}$ are uniformly distributed random numbers $0<\operatorname{ranf}<1$.

The self-consistency of the collisions of test and field particles can be improved by sampling field particles from a grid-based distribution function that incorporates more moments of the velocity distribution and represents the actual evolving velocity distribution in more detail. Recall that the traditional TA collision algorithm samples field particles from the set of particles present in a spatially localized domain of the plasma to allow for an evolving and arbitrary velocity distribution. Of course, the additional self-consistency and detail comes with an additional computational cost. In the collision algorithms using Langevin equations incorporating drag and diffusion, Rosenbluth potentials must be computed and recomputed in order to maintain self-consistency with an arbitrary distribution function that evolves $[4,15]$. The traditional and grid-based TA algorithms do not require calculation of the Rosenbluth potentials.

When the collisional scattering of the velocity vector in one time step is too large, neither grid-based nor binary collision algorithms are accurate. If only the scattering angle becomes too large in Eq.(1) or (2) when the time step is too large, the angular diffusion rate becomes inaccurate, but the change in the testparticle energy may still be small. We also find (Sec. III) that particle statistics influence the energy conservation. We note that when the time step becomes too large in the binary collision algorithm, conservation of energy and momentum is still preserved; and although relaxation rates may not be reproduced accurately, the binary collision algorithm fails gracefully.

To remedy the non-conservation of momentum and energy in the grid-based algorithm, after a likespecies collision one can shift the mean of the test-particle velocities in each dimension and in each cell, and scale the velocity relative to the mean velocity so that the momentum and kinetic energy are restored to their values before the collision [4,6]. The non-relativistic implementation for this is exact. For the relativistic case, we shift the mean momentum to conserve the total momentum in each cell. We then scale the momentum per unit mass relative to the local mean by a factor $(1+\alpha)$ and expand the expression for the kinetic energy for $|\alpha|<<1$, in order to solve for $\alpha$ such that kinetic energy conservation is restored

$$
m c^{2} \sum\left[1+\left((1+\alpha)\left(\frac{\mathbf{v}}{c}-\frac{\langle\mathbf{v}\rangle}{c}\right)+\frac{\langle\mathbf{v}\rangle}{c}\right)^{2}\right]^{1 / 2}-1 \approx m c^{2} \sum\left[1+\left(\frac{\mathbf{v}}{c}\right)^{2}\right]^{1 / 2}-1+\frac{\alpha \frac{\mathbf{v}}{c} \cdot\left(\frac{\mathbf{v}}{c}-\frac{\langle\mathbf{v}\rangle}{c}\right)}{\left[1+\left(\frac{\mathbf{v}}{c}\right)^{2}\right]^{1 / 2}}
$$


In Eq.(4) $\mathbf{v}$ is the momentum per unit mass. In practice, $|\alpha|$ is quite small, e.g., $|\alpha|=\mathrm{O}\left(10^{-5}-10^{-4}\right)$ for sufficiently small collisional scattering angle per time step. For a light species collisionally scattering on a heavy species, we shift and scale the heavy species particle velocities because this results in a smaller relative adjustment of the velocities.

In carrying out the computations for the grid-based TA collision algorithm as compared to the traditional TA algorithm we note the following characteristics. In the grid-based TA algorithm the additional gather/scatters from/to the grid are a small burden compared to the total computational burden of performing the collisions, as are the sorting into unique pairs and the optimized shuffling of the pairs [20] in the classic TA algorithm. The biggest difference in the computational burden between grid-based TA and basic TA algorithms is that $N_{\text {p,cell }}$ particles corresponding to $N_{\text {p,cell }}$ pairs are collided in the grid-based TA scheme, while there are only $N_{\mathrm{p}, \text { cell }} / 2$ pairs in the basic TA algorithm. Here we are assuming $N_{\mathrm{p}, \text { cell }}$ is even. This factor of two applies to both like-species and unlike-species collisions using the grid-based TA algorithm.

Timings of our implementation of the grid-based TA algorithm indicate that it is slightly slower than our implementation of the drag-diffusion Langevin equation collision algorithm of Lemons et al. [6] by a factor of $\sim 1.35$, but neither implementation has been optimized for speed. Our implementation of the shiftscale fix of the energy and momentum for the grid-based collision algorithms requires an additional 35\% of the computational time. Thus, the grid-based TA collision algorithm with shift-scale energy-momentum conservation is $\sim 2.7 \times$ the computational cost of the standard TA binary collision algorithm.

In Secs. III and IV we compare the grid-based TA algorithm to the Lemons algorithm for the relaxation of a weak temperature anisotropy and discuss the extension of the grid-based TA algorithm for a particular physical situation of interest in inertial confinement fusion.

\section{Example of using the grid-based Takizuka and Abe collision algorithm and comparison to a drag-diffusion Langevin equations algorithm}

In this section of the paper we report results of an example of using the grid-based Takizuka and Abe collision scheme for a particular relaxation problem and a discussion of performance characteristics. In [11] we experience was reported with both grid-based drag-diffusion and binary TA collison algorithms focusing on time-step considerations. Here we exercise the grid-based and traditional binary TA collision

algorithms for a small time step giving accurate results and examine some of the performance characteristics as a function of particle statistics. 
Here we also compare the results of the grid-based TA algorithm to results of the drag-diffusion Langevin equations algorithm introduced by Lemons et al. [6]. The Lemons et al. drag-diffusion equations are based on

$$
\begin{aligned}
& \Delta \mathrm{v}=F_{v} \Delta t+g(\mathrm{v}) \Delta t^{1 / 2} N_{1}, \quad g(\mathrm{v})=\left[\frac{A_{D}}{v} G\left(\frac{v}{\sqrt{2} \mathrm{v}_{t h, f}}\right)\right]^{1 / 2} \\
& F_{v}=-\left(A_{D} / 2 \mathrm{v}^{2}\right)\left[\left\{\left(1+\frac{m_{t}}{m_{f}}\right) \mathrm{v}^{2} / \mathrm{v}_{t h, f}^{2}+1\right\} G-\Phi\right], \\
& A_{D}=8 \pi n_{f} q_{t}^{2} q_{f}^{2} \ln \Lambda / m_{t}^{2} \\
& G(u) / u=[\Phi(u)-u d \Phi / d u] / 2 u^{3} \approx \frac{1}{2} \frac{1}{u^{3}+\frac{3 \sqrt{\pi}}{4}}, \quad \Phi=\operatorname{erf}(u), \\
& u=\mathrm{v}_{t} / \sqrt{2} \mathrm{v}_{t h, f}, \mathrm{v}_{t h, f}=\left(T_{f} / m_{f}\right)^{1 / 2} \\
& \Delta \theta=\left[\frac{A_{D}}{v^{3}}(\Phi-G)\right]^{1 / 2} \Delta t^{1 / 2} N_{2}, \Delta \phi=2 \pi U[0,1] \\
& N_{1,2} \equiv \text { Gaussian random nos., }<N_{i}>=0,<N_{i}^{2}>=1, \\
& U \equiv \text { uniform random no., } 0 \leq U \leq 1
\end{aligned}
$$

In Eq.(5) the drag-diffusion coefficients depend on the spatially local density, mean drift, and thermal velocity of the field particles accumulated on the spatial grid, which evolve self-consistently in time. For like-species collisions, the test and field particle velocity distributions are the same.

There are some features in the integration of Eq.(5) that deserve comment. As noted in [6], there is a divergence of some of the drag-diffusion coefficients at small speeds; and [6] provides a prescription for dealing with the divergence. A rare large kick in the polar angle $\Delta \theta$ in one time step can be tolerated if it is a rare event, because the angle can wrap around every $2 \pi$ radians without generating a significant energy error. However, a large kick in the speed can significantly spoil the energy moment of the total distribution function. In our implementation of the Lemons algorithm we include a clamp on the change of speed in one time step such that if $|\Delta \mathrm{v} / \mathrm{v}|>1, \Delta \mathrm{v}$ is set to zero. When this clamp is not present, occasionally unphysically large jumps in the energy moments of the particle distribution occur. Both TA and Lemons algorithms were implemented in a particle code $[11,21]$ where special relativity was included in the kinematics. In consequence, the procedure for imposing ad hoc energy-momentum conservation uses Eq.(3) and is approximate.

Figures 1-4 show the results of a series of particle simulations examining the performance of the gridbased Takizuka and Abe collision algorithm. The test case involved is the collisional relaxation of a weak temperature anisotropy $\left(T_{\mathrm{y}}=0.9 T_{\mathrm{x}}, T_{\mathrm{x}}=T_{\mathrm{z}}\right)$ in which we vary the number of particles per cell for fixed product of the characteristic collision frequency $v_{*}$ and the time step $\Delta t$ in one spatial dimension, where $v_{*}=(2 \sqrt{2}) v_{0}, v_{0}=\sqrt{2} \pi q^{4} n \ln \Lambda / \sqrt{m} T^{3 / 2}$ and $v_{0}$ is the Braginskii [22] characteristic collision frequency for like- 
species collisions. The value of $\ln \Lambda$ was scaled so that the value of $v_{*} \Delta t$ can be set artificially. The exponential relaxation rate for a weak temperature anisotropy is given by Trubnikov [9], $v_{\text {relax }}=(8 / 5 \sqrt{2 \pi}) v_{0}$.

In Figures 1 and 2 we plot the relaxation of the weak temperature anisotropy normalized to the initial anisotropy as a function of time and the asymptotic theory, $\exp \left(-v_{\text {relax }} t\right)$. In Figure 1 a we used $N_{\text {p,cell }}=400$ particles per cell and 100 cells, and there is good agreement among the three results. Figure 1a shows results for the relaxation of a weak temperature anisotropy by like-species collisions vs. time using the TA algorithm, the TA algorithm with sampling, and the TA algorithm with sampling and ad hoc energy and momentum conservation for $v_{*} \Delta t=0.01$ and 100 time steps. Figure $1 \mathrm{~b}$ shows the corresponding results obtained with the self-consistent Lemons et al algorithm and the Lemons algorithm with ad hoc energy and momentum conservation for the same time step as in Fig. 1a. The traditional TA algorithm results give the best agreement with theory in Fig. 1a. The agreement of the grid-based TA algorithm is slightly better than the two variants of the Lemons algorithm. Imposition of the ad hoc energy and momentum conservation does not degrade the agreement of the simulated relaxation rates with the theory. We vary the number of particles per cell in the results shown in Figure 2 for the grid-based TA and Lemons algorithms with $v_{*} \Delta t=0.01$. The agreement with the theoretical relaxation rate steadily improves with increasing particle statistics. We deem the agreement with respect to the theory to be good for greater than 200 particles per cell. The relative agreement with theory for the grid-based TA and Lemons algorithms is similar.

In Figures 3 and 4 we assess the conservation of energy in the grid-based TA and Lemons algorithms. Energy (and momentum) conservation improves with increasing numbers of particles per cell. The total kinetic energy of the simulation as a function of time for a series of simulations using the grid-based TA algorithm in which different numbers of particles per cell were used is plotted in Figure 3. In Figure 4 we show results for 400 particles per cell using the grid-based TA algorithm, the Lemons algorithm, and the Lemons algorithm with ad hoc energy-momentum conservation imposed. We note that the energy errors are similar for the grid-based TA and Lemons algorithms. The energy errors for the Lemons algorithm with energy-momentum conservation imposed are significantly improved, and the results reveal a very weak systematic growth of the kinetic energy. We note two issues that may affect the result shown in Fig. 4c: (1) the ad hoc energy-momentum conservation does not restore energy conservation exactly because of the use of Eq. (3) to cope with special relativity in the particle code; (2) there can be a noise-induced instability in the drag-diffusion Langevin equations when the drag-diffusion coefficients are calculated selfconsistently [23]. For the temperature dependence of the collision frequency in Coulomb collision rate $T$ $3 / 2$, the analysis in [23] predicts instability with a growth rate scaling inversely with the number of particles for $N_{\mathrm{p}}>>1$. Reference [23] notes that the instability can be nullified by shifting and scaling the velocities 
after each collision to exactly conserve energy or by constraining the random numbers used in the collisions to exactly conserve their mean and variance.

Two types of root-mean-square (RMS) error in the kinetic energy are plotted as a function of the number of particles per cell in Figure 5:

$$
\begin{aligned}
\text { RMS Err \# } 1 & \equiv\left[\sum_{i=1}^{N}(K E(i \Delta t) / K E(0)-1)^{2} / N\right]^{1 / 2} \\
\text { RMS Err \# } 2 & \equiv\left[\sum_{i=1}^{N}(K E(i \Delta t)-K E((i-1) \Delta t))^{2} / N \cdot K E(0)^{2}\right]^{1 / 2}
\end{aligned}
$$

where the sums are over the $N$ collision events for each particle and $K E$ is the sum of the kinetic energy over particles. The first RMS error is an average over the cumulative error in the energy, while the second RMS error is the average over kinetic energy errors at each time step. We observe that the asymptotic error scaling $1 / N_{\mathrm{p}, \text { cell }}^{1 / 2}$ emerges for the grid-based TA algorithm in Fig. 5a. When we repeated the same set of computations with the Lemons, et al collision algorithm, the RMS errors in Fig. 5b are similar in magnitude and also scale as $1 / N_{\mathrm{p}, \text { cell }}^{1 / 2}$.

Because restoring energy and momentum conservation after the collisions comes at a significant computational cost, it is important to understand the magnitude of the non-conservation problem. A simple estimate for the energy error in one time step (RMS Err \#2) in the grid-based TA scheme can be obtained as follows. In the grid-based TA scheme the relative velocity vector is scattered through a small angle on each time step. For the case of test and field particle velocity distribution functions at the same temperature, the scattering of test particle momenta through an angle should conserve total energy statistically if the sampling of the field particles, the random numbers used in the collisions, and the placement in phase space of the test-particle velocity vectors are random and uncorrelated, while symmetrically and uniformly distributed. However, the random numbers are not perfectly uniform and symmetric so that the standard error in any statistical quantity is $\mathrm{O}\left(1 / N_{\mathrm{p}}{ }^{1 / 2}\right)$; and the energy error is expected to scale as $\mathrm{O}\left(\left(v_{*} \Delta t\right)^{1 / 2} / N_{p}^{1 / 2}\right)$. We expect that the cumulative energy error (RMS Err \#1) will be larger by $[N(N+1) / 2]^{1 / 2} \sim 70$ for the $N=100$ collision events in the simulations.

The kinetic energy change in one time step in the Lemons scheme derives from a computation of $\Delta K E=\left\langle\frac{1}{2} m(\mathrm{v}+\Delta \mathrm{v})^{2}-\frac{1}{2} m \mathrm{v}^{2}\right\rangle=\left\langle m\left(\mathrm{v} \Delta \mathrm{v}+\frac{1}{2} \Delta \mathrm{v}^{2}\right)\right\rangle$ using Eq.(5) and then summing over the test particles, assuming the field particles have net zero mean drift. This leads to three terms to $\mathrm{O}(\Delta t)$, i.e., , 


$$
\Delta K E=\left\langle\int d^{3} \mathrm{v} f(\mathbf{v})\left[a(\mathrm{v}) \Delta t+b(\mathrm{v}) \Delta t\left(N_{1}^{2}-1\right)+c(\mathrm{v}) \Delta t^{1 / 2} N_{1}\right]\right\rangle
$$

where $a, b$, and $c$ are functions of the speed $\mathrm{v}$ and are derived from the drag-diffusion coefficients, $f(\mathbf{v})$ is the test-particle velocity distribution function, and the integral is represented by a sum over particles in the simulation. If the test-particle distribution function is the same as the Maxwellian distribution function used for the field particles, then the integrated contribution of the deterministic term involving $a$ analytically vanishes. However, this cancellation is not perfect in the simulation due to the statistical sampling of the distribution function; and the standard error in the cancellation is of relative order $1 / N_{\mathrm{p}}{ }^{1 / 2}$. Furthermore, the ensemble averages of the terms involving $b$ and $c$ also have standard errors of relative order $1 / N_{\mathrm{p}}{ }^{1 / 2}$. Furthermore, the functional dependence of the self-consistent drag-diffusion coefficients on the velocity moments of the velocity distribution can lead to noise-induced damping or growth of the energy as analyzed in Ref. [23]. Thus, the analysis of Eqs.(5), (6), and (7) is quite complicated. Nevertheless, we analytically compute the contribution to $\triangle K E$ from the standard error in the leading term in $\mathrm{O}\left(\Delta t^{1 / 2}\right)$, i.e., diffusion term $c(\mathrm{v}) \Delta t^{1 / 2}$ in Eq.(7), to obtain an estimate for the error in the kinetic energy: $\mathrm{O}\left(\left(v_{*} \Delta t\right)^{1 / 2} / N_{p}^{1 / 2}\right)$, which is the same as for the grid-based TA algorithm, if we assume that the noiseinduced instability, if present, is a weak effect. The results in Figs. 5a and $5 \mathrm{~b}$ are reasonably consistent with the estimates for RMS Err \#1 and \#2.

\section{Discussion}

We see from the performance of the grid-based TA algorithm that the grid-based algorithm requires twice the computation of the standard binary TA algorithm. With energy-momentum conservation imposed, the grid-based TA algorithm results in more than $2-3 \times$ the computational burden of the traditional TA algorithm in our implementation. The grid-based TA algorithm is a little slower than our implementation of the Lemons algorithm and is at least as accurate for the same time step and number of particles. What then might be the advantages of the grid-based TA algorithm compared to other collision algorithms?

As part of a comparison of collision algorithms extending beyond the results reported in [11], we undertook a series of simulations varying the size of the collisional time step and studying the same relaxation of a weak temperature anisotropy problem addressed here. We studied relaxation driven by electron-electron collisions using both the Lemons, et al. grid-based algorithm [6] and the traditional TA binary collision algorithm[1]. Both algorithms yield good results compared to theory for $v_{*} \Delta t \leq 0.25$ Separately we studied the same relaxation problem with only electron-ion collisions using the two 
algorithms. By using the TA binary algorithm for electron-ion (hydrogen) collisional relaxation of an electron temperature anisotropy, we were able to employ a time step that is $\mathrm{O}(10 \times)$ of those allowed in using the Lemons et al. algorithm [6]. This finding we attribute to having to resolve the diffusive velocity step in the perpendicular velocity diffusion at low velocities in the Lemons et al algorithm given the unfavorable scaling with $\left(\mathrm{m}_{\mathrm{i}} / \mathrm{m}_{\mathrm{e}}\right)^{1 / 2}$ of the perpendicular velocity diffusion rate for electron-ion collisions compared to the diffusion rate for electron-electron collisions, as explained in [11]. Thus, the TA collision algorithm allows the use of a substantially larger time step for electron-ion collisions than does the Lemons, et al algorithm without modification. (However, if the Lemons, et al. algorithm for electron-ion collisions is reduced to just pitch-angle collisions with the drag and diffusion of the electron speed suppressed, the studies in [11] demonstrate that time steps as big as those used with the TA algorithm can be used with similarly good accuracy obtained.) We also note that, as stated earlier, the drag-diffusion algorithms [3, 4, 5,6 ] can be vulnerable to noise-induced instability [23]; and the TA algorithm is more tolerant with respect to a rare large kick than is the Lemons et al algorithm unless some clamp or limiter on the magnitude of velocity kicks is installed in the latter.

The grid-based TA algorithm is straightforwardly extended to accommodate additional physics affecting the collision model, e.g., the stopping and scattering of relativistic electron beams in dense plasmas, as in the model of Atzeni, Schiavi, and Davies [24]. This collision model is of interest in the study of inertial confinement fusion involving fast ignition. Atzeni, et al. derive the variance of the polar scattering angle in the laboratory frame for relativistic electrons collisionally scattering on plasma electrons using the Möller cross-section and including screening:

$$
\left.\frac{\left\langle\Delta \theta^{2}\right\rangle}{\Delta t}\right|_{e-e}=\frac{8 \pi e^{4} n_{e}}{\gamma^{2} m_{e}^{2} c^{2} \mathrm{v}}\left[\ln \Lambda-\frac{\ln [2(\gamma+3)+1}{2}\right]
$$

and relativistic electrons collisionally scattering on plasma ions including screening

$$
\left.\frac{\left\langle\Delta \theta^{2}\right\rangle}{\Delta t}\right|_{e-i}=\frac{8 \pi e^{4} Z^{2} n_{i}}{\gamma^{2} m_{e}^{2} c^{2} \mathrm{v}}\left[\ln \Lambda-\frac{1+\mathrm{v}^{2} / c^{2}}{2}\right]
$$

where $\gamma$ is the relativistic factor for the test particle with velocity $\mathrm{v}$ and $\ln \Lambda=4 \pi \lambda_{D e} / \lambda_{d B}=4 \pi \lambda_{D e} m_{e} c \beta \gamma / h$ where $\lambda_{\text {De }}$ is the electron Debye length, $h$ is Planck's constant, $\lambda_{\mathrm{dB}}$ is the deBroglie wavelength, and $\beta=\mathrm{v} / c$ in the physics model of Atzeni et al [24]. The plasma electrons are assumed to be non-relativistic and essentially stationary compared to the relativistic electrons. Note that the variances of the deflection angles in Eqs.(8) and (9) are calculated in the laboratory frame and a 
typographical error in the exponent of $\gamma$ in the denominator in Eq.(24) of [24] has been corrected here. We also note that the pre-factors in Eqs.(8) and (9) are consistent with those in the semi-relativistic model in Eq.(2) with the replacement for the relativistic electron test-particle momentum in the denominator: $p=\gamma m_{\mathrm{e}} \mathrm{v} \approx \gamma m_{\mathrm{e}} \mathrm{c}$, assuming the velocity of the field particle is negligible compared to the relativistic electron in the laboratory frame. Equations (8) and (9) represent a computation of corrections to $\ln \Lambda$. In the Appendix we give a summary of the relativistic extension to the TA algorithm for colliding a pair of particiles.

A model for collisions when a minority component of relativistic electrons is present could use Eqs.(8) and (9), and the grid-based TA algorithm for the relativistic electrons scattering on the background plasma; and the inter- or intra-species collisions of the non-relativistic electron and ion background particles could use the traditional TA, grid-based TA, or Lemons et al algorithms. In this model, it is assumed that the scattering of the relativistic electrons off other relativistic electrons is negligible compared to the scattering off the background electrons and ions, and that the scattering of the background electrons and ions off the relativistic electrons is negligible compared to the scattering off the background electrons and ions.

In the simulation model of Sentoku and Kemp [16] unequally weighted particles are introduced to represent a large range in plasma density spanning many orders of magnitude. Sentoku and Kemp [16] extend the TA/Nanbu binary collision model to accommodate unequally weighted particles, but energymomentum conservation can no longer be preserved. The grid-based version of the TA/Nanbu collision algorithm is easily applied to the use of unequally weighted particles, and energy-momentum conservation can be restored by shifting and scaling the momenta after the collision as described earlier.

In summary, the grid-based TA collision algorithm should be considered as a good alternative to gridbased collision algorithms like those in [3], [4], [5] and [6]. Like other grid-based collision algorithms, the grid-based TA scheme is well suited for including Coulomb collisions in a particle-fluid hybrid plasma simulation. Its performance is at least as good or better than the grid-based algorithms in [4], [5] and [6], is more tolerant to the rare large Gaussian random number, is not subject to a possible noise-induced numerical instability, and allows the use of a significantly larger time step for electron-ion collisions. Furthermore, as suggested in Sec. II, the detailed self-consistency of the field particle velocity distribution function with the evolving kinetic plasma and its influence on the collisional relaxation can be accommodated in the grid-based TA scheme in principle without having to calculate Rosenbluth potentials. Finally, the grid-based TA collision algorithm can be extended straightforwardly to different collision models. 


\section{Acknowledgment}

We are grateful to Alex Friedman, Andreas Kemp, David Larson, Mark Rosin, and Russ Caflisch for useful discussions, suggestions, and encouragement.

\section{Appendix: Relativistic Binary Collisions}

Here we give a summary of the steps in the semi-relativistic generalization of the TA collision algorithm. The analysis involves Lorentz transformation of the momentum vectors of the two colliding particles between three frames of reference: laboratory frame $(L)$, rest frame of the field (second) particle $(R)$, and center-of-mass frame $(C M)$. One first designates a test (1) and field (2) particle pair, and Lorentz transforms to the rest frame of the field particle which is defined by

$$
\gamma_{R}\left(\mathbf{p}_{2}^{L}-\frac{\mathbf{V}_{R}}{c^{2}} E_{2}^{L}\right)=0, \quad \gamma_{R}=\left(1-\frac{\mathbf{V}_{R}^{2}}{c^{2}}\right)^{-1 / 2}, \quad E_{2}^{L}=\left(p_{2}^{L^{2}} c^{2}+m_{2}^{2} c^{4}\right)^{1 / 2}
$$

The test-particle momentum $\boldsymbol{p}_{1}{ }^{\mathrm{L}}$ is Lorentz transformed to the rest frame $R$ using the $\boldsymbol{V}_{\mathrm{R}}$ determined in Eq.(A1). The momentum vector $\boldsymbol{p}_{1}{ }^{\mathrm{R}}$ is then represented with respect to rotated Cartesian axes such that the new $z_{\mathrm{R}}{ }^{\prime}$ axis is aligned with $\boldsymbol{p}_{1}{ }^{\mathrm{R}}$ as rotated in Eq.(2) of [1] and Eq.(23) of [6]. In the rest frame $\boldsymbol{p}_{2}{ }^{\mathrm{R}}=0$ and after the collision event, the scattered test particle momentum $\boldsymbol{q}_{1}{ }^{\mathrm{R}}$ will make a polar angle $\Delta \theta$ with the $z_{\mathrm{R}}$ ' axis determined with $\Delta \theta$ determined in Monte Carlo fashion using Eq.(2), (8), or (9), for example.

To compute the magnitudes and directions of the scattered momenta $\boldsymbol{q}_{1}{ }^{\mathrm{R}}$ and $\boldsymbol{q}_{2}{ }^{\mathrm{R}}$, we need to consider the collision in the center-of-mass frame $C M$. In the $C M$ frame the momenta of the test and field particles must sum to zero, by definition. Thus, the test and field momenta are equal and oppositely directed both before and after the collision, and are simply rotated by the small angle $\alpha$ in the scattering plane due to the collision. The collision also introduces a completely random azimuthal angle with respect to the $z_{\mathrm{R}}$ ' axis. Moreover, the magnitudes of the momenta vectors in the $C M$ frame are unchanged by the collision in order to conserve energy either non-relativistically or relativistically if the collision is elastic (e.g., ignoring radiation losses and assuming that the collision is non-ionizing). By considering the Lorentz transformations from the $R$ frame to the $C M$ frame with the condition that $\boldsymbol{p}_{2}{ }^{\mathrm{cm}}=-\boldsymbol{p}_{1}^{\mathrm{cm}}$, one readily determines

$$
\begin{aligned}
& p_{1 z}^{c m}=\gamma_{c} p_{1 z}^{R} m_{2} c^{2} /\left(m_{2} c^{2}+E_{1}^{R}\right), \\
& V_{c}=p_{1 z}^{R} /\left(m_{2}+E_{1}^{R} / c^{2}\right), \gamma_{c}=1 /\left(1-V_{c}^{2} / c^{2}\right)^{1 / 2}
\end{aligned}
$$

where $V_{\mathrm{c}}$ is the velocity of the $C M$ frame with respect to the rest frame $R$. Rotations and a Lorentz transformation using Eq.(A1) determines $p_{1 z}{ }^{\mathrm{R}}$ from $\boldsymbol{p}_{1}{ }^{\mathrm{L}}$. We can now determine the angle $\alpha$ by Lorentz 
transforming $\boldsymbol{q}_{1}{ }^{\mathrm{CM}}$ back to frame $R$ and by using the vector components of $\boldsymbol{q}_{1}{ }^{\mathrm{R}}$ to evaluate $\tan \Delta \theta$, where $\Delta \theta$ is determined by the product of a Gaussian random number and the square root of the variance $<\Delta \theta^{2}>$ accrued in one time step $\Delta t$ calculated from Eq.(2), (8), or (9), for example. Then

$$
\begin{aligned}
& q_{1 \perp}^{R}=p_{1}^{c m} \sin \alpha, q_{1 z}^{R}=\gamma_{c}\left(p_{1}^{c m} \cos \alpha+\frac{V_{c} E_{1}^{c m}}{c^{2}}\right), \\
& \tan \Delta \theta=\frac{q_{1 \perp}^{R}}{q_{1 z}^{R}}=\frac{p_{1}^{c m} \sin \alpha}{\gamma_{c}\left(p_{1}^{c m} \cos \alpha+\frac{V_{c} E_{1}^{c m}}{c^{2}}\right)}=\frac{\sin \alpha}{\gamma_{c}\left(\cos \alpha+\frac{E_{1}^{c m}}{\gamma_{c} m_{2} c^{2}}\right)}
\end{aligned}
$$

From Eq.(A3) one determines $\sin \alpha$ and then $\boldsymbol{q}_{1}{ }^{\mathrm{R}}$, keeping in mind that the theory assumes small-angle scattering. It is then straightforward to evaluate $\boldsymbol{q}_{2}{ }^{\mathrm{R}}$ by using $\boldsymbol{q}_{2}{ }^{\mathrm{CM}}=-\boldsymbol{q}_{1}{ }^{\mathrm{CM}}$ and performing the Lorentz transformation from $C M$ to $R$. The post-collision random azimuthal angle $\phi$ can be introduced in the $C M$ frame, which leads to $\left(q_{1 \mathrm{x}}{ }^{\mathrm{R}}, q_{1 \mathrm{y}}{ }^{\mathrm{R}}\right)=q_{1_{\perp}}{ }^{\mathrm{R}}(\cos \phi, \sin \phi)$. We next reverse the rotation of axes performed in the rest frame $R$ and finally use the inverse of the Lorentz transformation defined in Eq.(A1) to determine the post-collision momenta $\boldsymbol{q}_{1}{ }^{\mathrm{L}}$ and $\boldsymbol{q}_{2}{ }^{\mathrm{L}}$.

In the non-relativistic limit, Eqs.(A2) and (A3) lead to

$$
\begin{aligned}
& \sin \Delta \theta=\frac{\sin \alpha}{1+\frac{m_{1}}{m_{2}}}, p_{1}^{C M}=m_{12}\left(p_{1 z}^{L} / m_{1}-p_{2 z}^{L} / m_{2}\right)=m_{12} u \\
& q_{1 \perp}^{L}=q_{1 \perp}^{R}=-q_{2 \perp}^{L}=-q_{2 \perp}^{R}=m_{12} u \sin \alpha \\
& q_{1 z}^{L}=m_{1} \mathrm{v}_{1 \mathrm{z}}^{L}-m_{12} u(1-\cos \alpha), q_{21 z}^{L}=m_{1} \mathrm{v}_{2 \mathrm{z}}^{L}+m_{12} u(1-\cos \alpha)
\end{aligned}
$$

where $u=\mathrm{v}_{1 \mathrm{z}}{ }^{\mathrm{L}}-\mathrm{v}_{2 \mathrm{z}} \mathrm{L}$. Hence, $\mathbf{q}_{1,2}^{L}=\mathbf{p}_{1,2}^{L} \pm m_{12} \Delta \mathbf{u}$, where $\Delta \mathbf{u}=\left[u_{z}, u_{\perp}\right]=[u(1-\cos \alpha), u \sin \alpha]$. These results recover the results of TA as in Eq.(1). We also note that for like-species collisions, $m_{12}=m_{1} / 2$ and the energy of the test particle after the collision in the rest frame $R$ is reduced by the collision, $\frac{1}{2 m_{1}}\left(q_{1 z}^{R^{2}}+q_{1 \perp}^{R^{2}}\right) \approx \frac{1}{2 m_{1}} p_{1 z}^{R^{2}}\left(1-(\sin \theta)^{2}\right)$, while the energy of the field particles increases. For $m_{1} / m_{2}<<1$, as in electron-ion collisions, $m_{12} \approx m_{1}$ and the energy of the test particle after the collision in the rest frame $R$ is unchanged to good approximation, $\frac{1}{2 m_{1}}\left(q_{1 z}^{R^{2}}+q_{1 \perp}^{R^{2}}\right) \approx \frac{1}{2 m_{1}} p_{1 z}^{R^{2}}$. 


\section{References}

[1] T. Takizuka and H. Abe, "A binary collision model for plasma simulation with a particle code," J. Comput. Phys. 25, 205 (1977).

[2] K. Nanbu, “Theory of cumulative small-angle collisions in plasmas,” Phys. Rev. E 55, 4642 (1997).

[3] M. E. Jones, D. S. Lemons, R. J. Mason, V. A. Thomas, and D. Winske, “A Grid-Based Coulomb Collision Model for PIC Codes,” J. Comput. Phys. 123169 (1996).

[4] W. M. Manheimer, M. Lampe, G. Joyce, "Langevin representation of Coulomb collisions in PIC simulations," J. Comput. Phys. 138, 563 (1997).

[5] B. I. Cohen, L. Divol, A. B. Langdon, and E. A. Williams, "Effects of Ion-ion collisions and inhomogeneity in two-dimensional kinetic ion simulations of stimulated Brillouin backscattering," Phys. Plasmas 13, 022705 (2006).

[6] D. S. Lemons, D. Winske, W. Daughton, B. Albright, "Small-angle Coulomb collison model for particle-in-cell simulations," J. Comput. Phys. 228, 1391 (2009).

[7] M. Sherlock, “A Monte-Carlo method for Coulomb collisions in hybrid plasma models," J. Comput. Phys. 227, 2286 (2008).

[8] L. Spitzer, The Physics of Fully Ionized Gases, Wiley Interscience, (New York,1956).

[9] B. A. Trubnikov, "Particle interactions in a fully ionized plasma," in Reviews of Plasma Physics (Consultants Bureau, New York NY, 1965), Vol. 1, 105 (1965).

[10]T. D. Rognlien and T. A. Cutler, "Transition from Pastukhov to collisional confinement in a magnetic and electrostatic well," Nuc. Fusion 20, 1003 (1980).

[11]B. I. Cohen, A. M. Dimits, A. Friedman, and R. E. Caflisch, IEEE Trans Plas Sci 38, 2394 (2010).

[12]C. M. Wang, T. Lin, R. Caflisch, B. I. Cohen, A. M. Dimits, "Particle simulation of Coulomb collisions: comparing the methods of Takizuka and Abe, and Nanbu," J. Comp. Phys. 227, 4308 (2008).

[13]A. M. Dimits, C. Wang, R. Caflisch, B. I. Cohen, and Y. Huang, "Calculation of Trubnikov and Nanbu collision kernels: implications for numerical modeling of Coulomb collisions," J. Comput. Phys. 228, 4881-4892 (2009).

[14]P. E. Kloeden and E. Platen, Numerical Solution of Stochastic Differential Equations, Springer-Verlag (Berlin, 1999), Sec. 10.3

[15]M. N. Rosenbluth, W. M. Macdonald, and D. L. Judd, "Fokker-Planck equation for an inverse-square force," Phys. Rev. 107, 1 (1957).

[16]Y. Sentoku, and A. J. Kemp, J. Comp. Phys. 227,6846 (2008).

[17]C. F. F. Karney, Comp. Phys. Rep. 4, 183 (1986); B. Braams and C. F. F. Karney, Phys. Fluids B 1, 1355 (1989). 
[18]J. D. Jackson. (1962). Classical Electrodynamics ( $1^{\text {st }}$ ed.). New York: John Wiley \& Sons. Chapts. 12 and 13.

[19]C. K. Birdsall and A. B. Langdon. (1985). Plasma Physics Via Computer Simulation. (McGraw-Hill, New York).

[20]R. A. Fisher, and F. Yates, F. (1948) [1938]. Statistical tables for biological, agricultural and medical research (3rd ed.). London: Oliver \& Boyd. pp. 26-27

[21]R. J. Procassini, Ph.D. thesis, University of California, Berkeley, 1990.

[22]S. I. Braginskii, "Transport processes in a plasma," in Reviews of Plasma Physics (Consultants Bureau, New York NY, 1965), Vol. 1, 205 (1965).

[23]D. S. Lemons, J. Lackman, M. E. Jones, and D. Winske, Phys. Rev. E 52, 6855 (1995).

[24]S. Atzeni, A. Schiavi, and J. R. Davies, Plasma Phys. Control. Fusion 51, 015016 (2009) 
Fig. 1
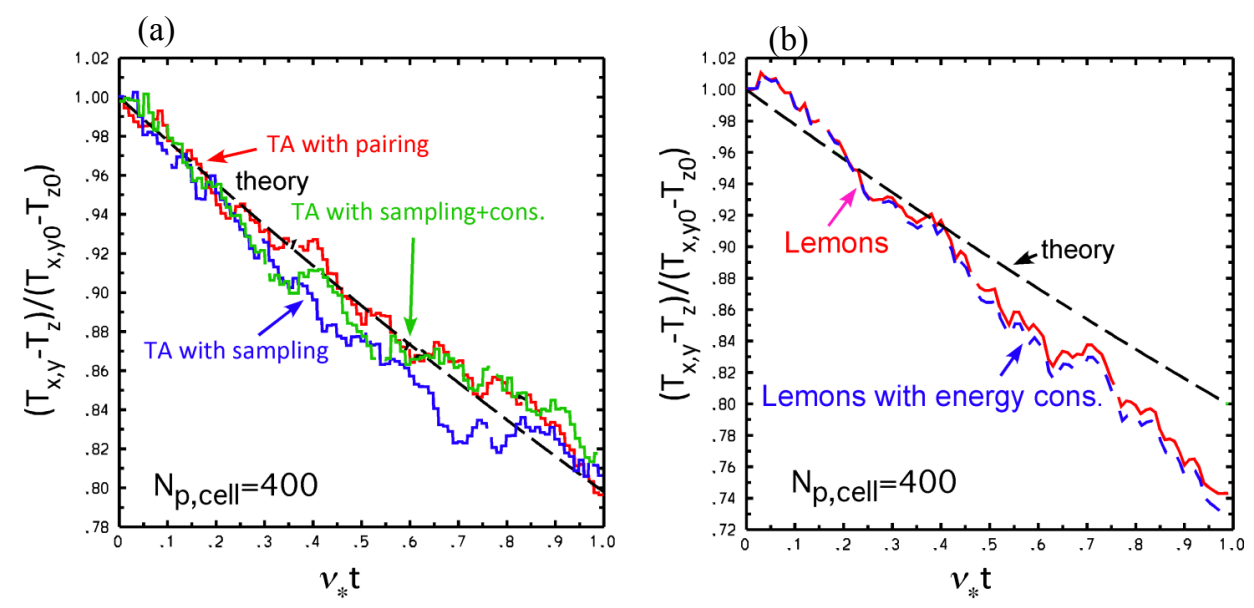

Fig. 1. Collisional relaxation (e-e collisions) of a $10 \%$ initial temperature anisotropy with $N_{\text {cell }}=400,40 \mathrm{~K}$ particles total, with $v_{*} \Delta t=v_{\mathrm{ee}, \mathrm{NRL}} \Delta t=0.01$ (a) Takizuka-Abe algorithms. (b) Lemons algorithm with and without energy conservation 
Fig. 2

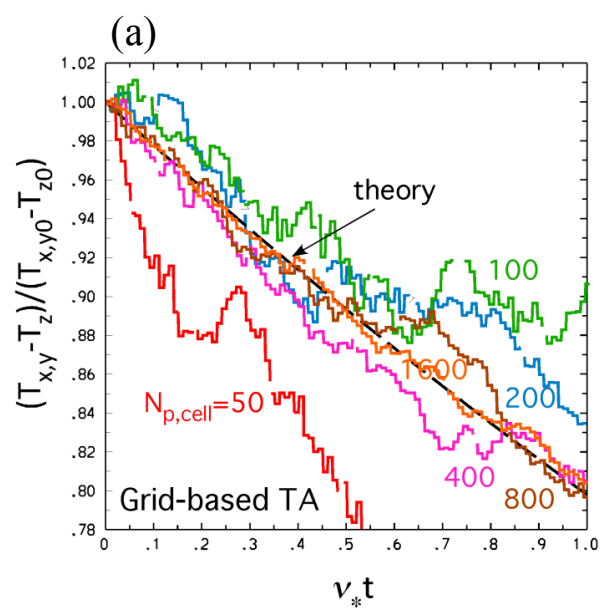

(b)

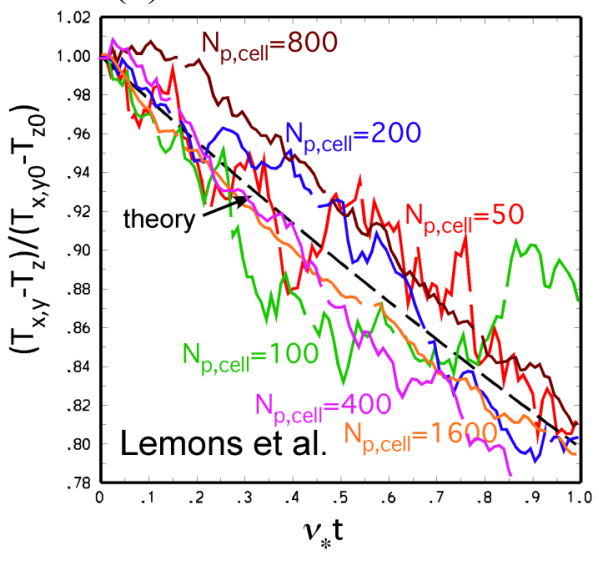

Fig. 2. Agreement of the (a) grid-based Takizuka-Abe and (b) Lemons et al collision algorithms for relaxation of a $10 \%$ temperature anisotropy compared to theory improves with more particles per cell. $v * \Delta t$ $=v_{\mathrm{ee}, \mathrm{NRL}} \Delta t=0.01$ 
Fig. 3
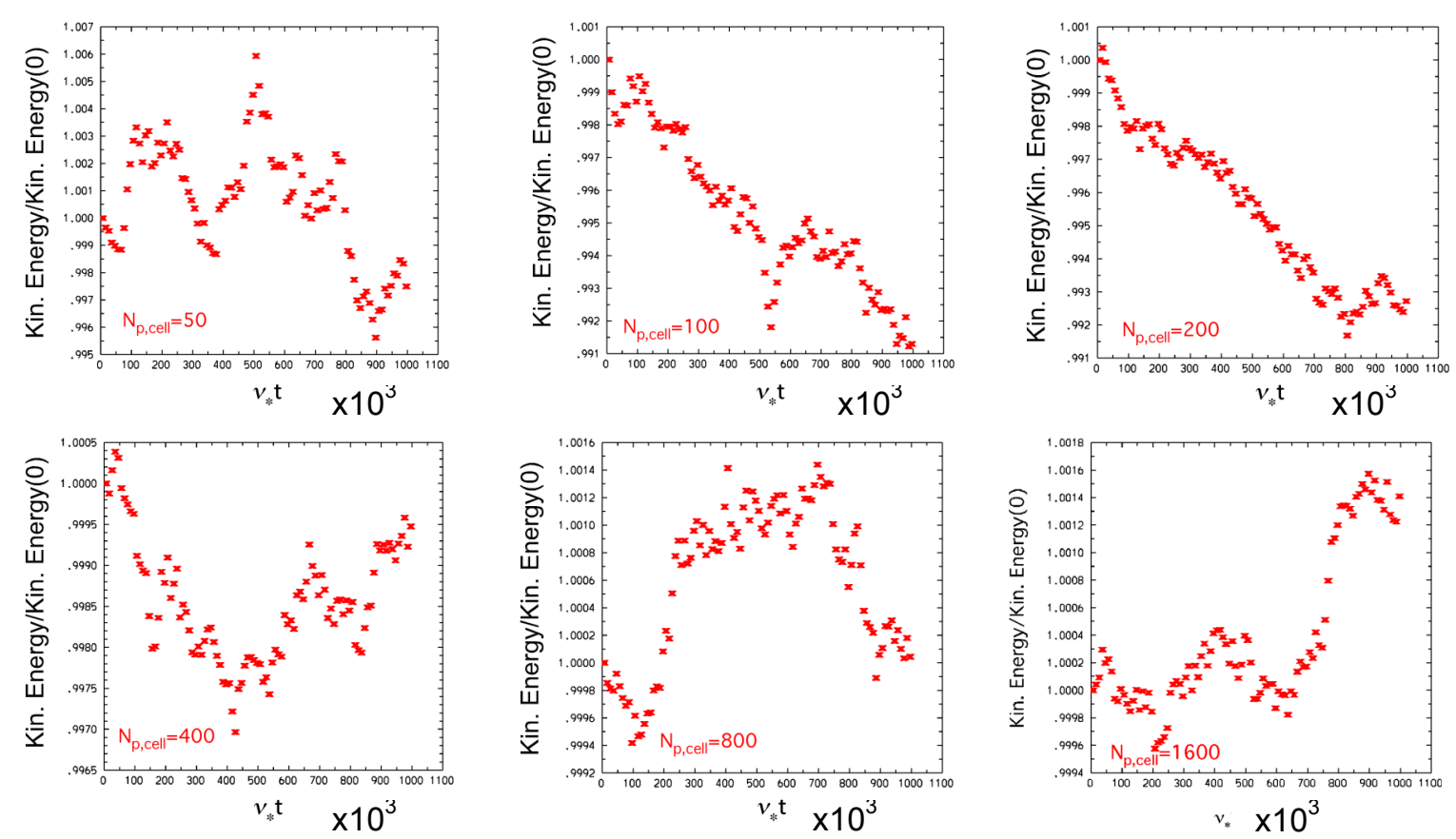

Fig. 3. Collisional relaxation of a weak temperature anisotropy using the grid-based TA algorithm. Kinetic energy vs. time as a function of increasing numbers of particles per cell. The kinetic conservation error decreases with increasing particles per cell $N_{\mathrm{p}, \text { cell }}$ 
Fig. 4

(a)

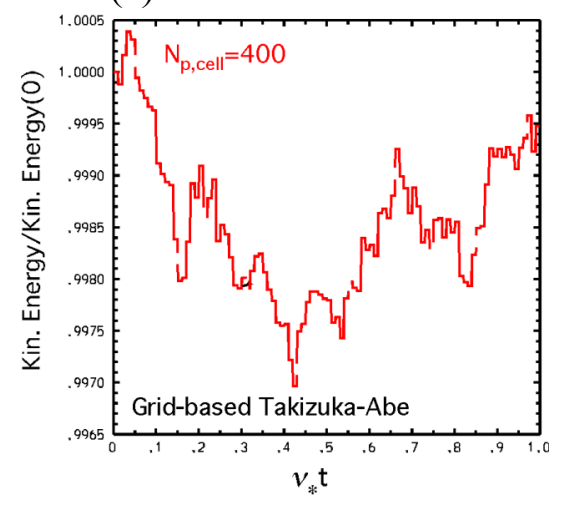

(b)

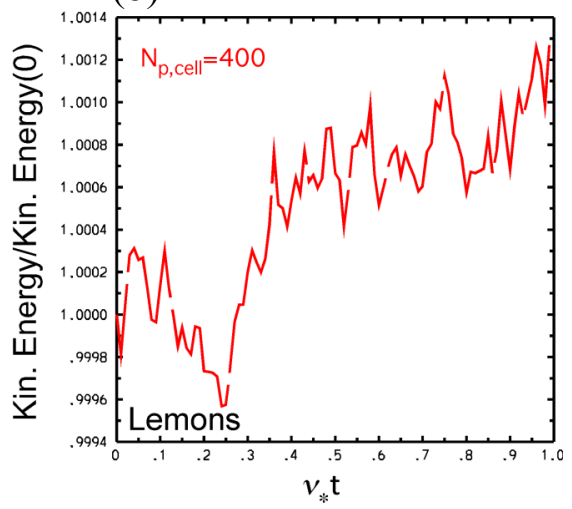

(c)

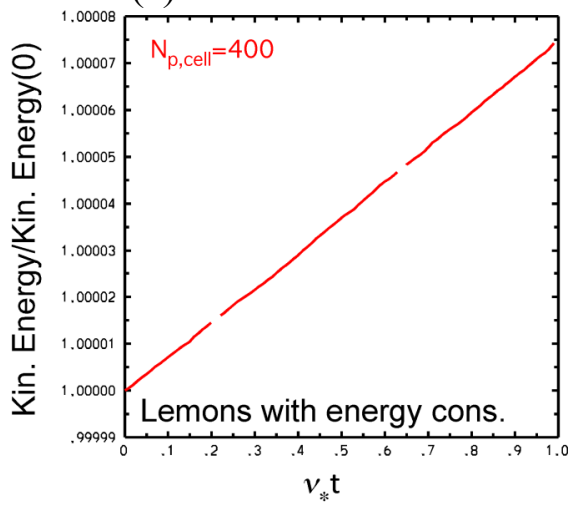

Fig. 4. Collisional relaxation of a weak temperature anisotropy. Kinetic energy normalized to the initital energy is plotted vs. time. (a) Takizuka-Abe grid-based algorithm. (b) Lemons et al. algorithm with selfconsistent drift and drag-diffusion coefficients. (c) Lemons et al algorithm with approximate energy conservation 
Fig. 5
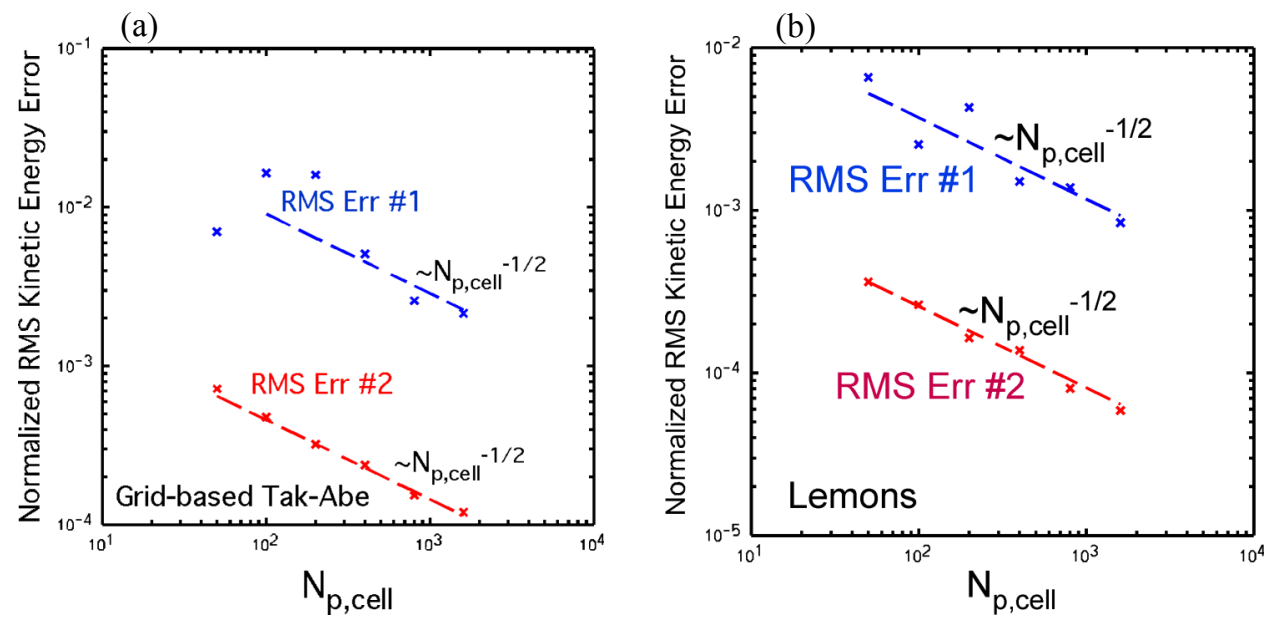

Fig. 5. Collisional relaxation of a weak temperature anisotropy. The root-mean-square (RMS) energy conservation errors decrease with increasing particles per cell. (a) Takizuka-Abe grid-based algorithm. (b) Lemons et al. algorithm. 